\title{
The Correlation Argument for Reductionism
}

\author{
Christopher Clarke*†
}

Reductionists say things like all mental properties are physical properties; all normative properties are natural properties. I argue that the only way to resist reductionism is to deny that causation is difference making (thus making the epistemology of causation a mystery) or to deny that properties are individuated by their causal powers (thus making properties a mystery); that is to say, unless one is happy to deny supervenience or to trivialize the debate over reductionism. To show this, I argue that if properties are individuated by their causal powers, then, surprisingly, properties are individuated by necessary coexemplification.

1. Property Monism versus Property Dualism. Some properties are social properties such as is the president of Colombia; some properties are mental properties such as likes broccoli; some properties are biological properties such as has a heart or is a mammal; some properties are physical properties such as has a high temperature; and indeed some properties are microphysical properties such as contains ammonium ions. Or so it is often said. But what is the relationship between these various domains of properties? Are all social properties in some sense mental or biological properties (Mellor 1982; Ruben 1984; Pettit 1993; Sawyer 2002)? Are all mental properties in some sense biological or physical properties (Smart 1959; Lewis 1966;

Received August 2017; revised April 2018.

*To contact the author, please write to: Faculty of Philosophy, Erasmus University Rotterdam, Postbus 1738, 3000 DR Rotterdam, Netherlands; e-mail: clarke@fwb.eur.nl; or Centre for Research in the Arts, Social Sciences, and Humanities, University of Cambridge, 7 West Road, CB3 9DP, United Kingdom; e-mail: cjc84@cam.ac.uk.

$\dagger$ Thank you Adrian Boutel, Christopher Cowie, Hugh Mellor, and Mark Pindar for your comments on an ancestor of this manuscript; and also to several anonymous referees for your generous and constructive suggestions. This work has received funding from the European Research Council under the European Union's Horizon 2020 research and innovation program (grant agreement 715530).

Philosophy of Science, 86 (January 2019) pp. 76-97. 0031-8248/2019/8601-0004\$10.00

Copyright 2019 by the Philosophy of Science Association. All rights reserved. 
Chalmers 1996)? Are all biological properties in some sense physical properties (Macdonald 1992; Rosenberg 2006)? Are all physical properties microphysical properties (Pettit 1993; Papineau 2001; Hüttemann 2004; Mellor 2008)? For that matter, are all normative properties in some sense natural properties (Streumer 2008)?

Thus for any two domains of properties one can ask, is one domain a subdomain of the other? Property monists say yes, and property dualists say no. Of course, these are relative designations: one might be a monist about social properties in relation to mental properties, for example, but a dualist about biological properties in relation to physical properties. (Another complication: so-called nonreductive physicalists are monists about all properties in relation to 'broadly physical' properties, but they are dualists about all properties in relation to 'narrowly physical' properties. ${ }^{1}$ And so, in examining an argument against all varieties of dualism, this article will be examining an argument against nonreductive physicalism.)

Claims of monism and dualism often figure as key premises or considerations in debates about laws and explanations in biology, psychology, and social science. What is the nature of biological, mental, and social scientific laws? Can the laws and facts of one domain be fully explained by invoking the laws and facts of another? Can explanations that invoke concepts from one domain nevertheless be reformulated solely in terms of the concepts of another, and without loss of understanding (Clarke 2016, 2017)? Although this article will not itself explore these questions about laws and explanations, the importance of these questions is part of what makes the issue of monism versus dualism so important itself. (Nevertheless, one ought to keep all these questions separate. To do so, I will talk about property monism vs. property dualism rather than about reductionism vs. antireductionism. The reason is that talk of reductionism vs. antireductionism blurs together the above questions about laws and explanations with the present question about properties.)

Within the philosophical literature, one finds three arguments that aim to establish monism over dualism. First, there are those arguments that appeal to Kim's infamous exclusion principle, or something similar such as filler functionalism. ${ }^{2}$ This article will not address such arguments. Second, there

1. A property is broadly physical if the property is 'determined' or 'realized' by the narrowly physical properties (Pereboom and Kornblith 1991; Shoemaker 2001). What counts as a narrowly physical property is, however, notoriously difficult to define (Ney 2008).

2. See Kim (1998, sec. 2) for explicit exposition. See Lewis $(1966,1994)$ and Jackson (2002) for a variant that appeals also to filler functionalism and that implicitly invokes the exclusion principle. See Bennett (2003) and Macdonald and Macdonald (2006) for critical responses. 
are the arguments that appeal to Kim's inheritance principle. ${ }^{3}$ This article will also not address such arguments. Third, there are various arguments that I will call correlation arguments. ${ }^{4}$ And one of the most interesting versions of the correlation argument is that given by Antony (2008).

The two most prominent premises of Antony's version of the correlation argument are the premise (Necess), which says that the properties of the first domain in question 'supervene' on the properties of the other domain in question, and, second, the premise (Indiv by Necess), which says that properties are individuated by 'necessary coexemplification' (sec. 2). Many philosophers warmly embrace Necess, many dualists included. But the status of Indiv by Necess is less clear (sec. 3). So the correlation argument, as it stands, is not as forceful as it might be.

This article aims to make the correlation argument more incisive and powerful and, thereby, to clarify the fundamental points that are at issue between monists and dualists. Section 2 gives my preferred version of the correlation argument, a version that comprehensively lays bare all the argument's commitments. In particular, I show how the argument can remain neutral on the existence and objectivity of multiply realizable properties and of disjunctive properties. Sections 3 and 4 will then show that Indiv by Necess follows from some broadly appealing theses about the metaphysics of causation and of properties. The most prominent of these theses are (Indiv by Powers): properties are individuated by their causal powers, and (Difference Making): singular causation is difference making. Section 5 shows that another assumption of the correlation argument follows from a plausible thesis about the semantics of 'the property of' locutions. Section 6 summarizes the achievement: there are four main ways in which a dualist can resist the correlation argument. Dualists can give up at least one of three metaphysical theses - Indiv by Powers, Difference Making, or Necesseach of which currently enjoys widespread acceptance among dualists. Or dualists can make their disagreement with monists a superficial or semantic disagreement: over what properties to label as physical, for example. Section 7 reviews the costs of each of these options.

Thus I hope to deepen our understanding of dualism's prospects and to push the debate forward by putting pressure on each dualist to be explicit

3. See $\operatorname{Kim}(1998,107-11)$ for an exposition and defense of the argument itself. Refer also to Kim (1992), Lewis (1994), Shapiro (2000), Heil (2003), and Heil and Robb (2003). See Pereboom and Kornblith (1991), Shoemaker (2001), and Baker (2007, 115-17) for critical responses.

4. See Smart (1959) and McLaughlin (2007). Bacon (1986), Kim (1992), Clapp (2001), and perhaps Jaworski (2002) may also be interpreted as offering a variant on this argument. Some correlation arguments appeal to considerations of simplicity (Smart 1959) or inference to the best explanation (McLaughlin 2007) to argue that it is rare for two distinct properties to be necessarily coexemplified. 
about which one of these four ways of resisting the correlation argument she will opt for.

2. The Correlation Argument. What is the most compelling version of the correlation argument? Let us begin with a very sketchy example of a correlation argument in action.

The following holds necessarily: for any given object, that object is alkaline if and only if it contains hydroxide ions or it contains ammonium ions. Or so I will pretend for ease of illustration. But, just for the moment, let us assume that the concept is alkaline succeeds in denoting a property. From this one infers that there also exists a property contains hydroxide or ammonium ions. But, one contends, this property counts as a microphysical property. So the property is alkaline is necessarily coexemplified with the microphysical property contains hydroxide or ammonium ions. (By saying that a property $A$ and a property $B$ are necessarily coexemplified, I mean that it is absolutely impossible for any object to exemplify property $A$ without that object also simultaneously exemplifying property $B$, and it is absolutely impossible for any object to exemplify property $B$ without that object also simultaneously exemplifying property $A$.) From this one infers that the property is alkaline is the very same thing as the microphysical property contains hydroxide or ammonium ions. The property is alkaline therefore counts as microphysical. One can now discharge the temporary assumption that the concept is alkaline denotes a property. This yields: if the concept is alkaline denotes any property at all, then the property it denotes counts as microphysical.

Although the above argument is extremely sketchy, it does give a rough illustration of my preferred version of the correlation argument, as applied to the concept is alkaline, and in relation to the domain of microphysical properties. I will now make the argument's assumptions fully explicit, and I will restate the argument in its general form, for arbitrary concept $a$, and in relation to an arbitrary domain of properties on which concept $a$ is assumed to supervene.

Consider those properties $S_{1}, S_{2}, S_{3}, \ldots$ that belong to the arbitrary domain of properties $\mathbb{S}$ in which we are taking an interest. This might be the domain of microphysical properties, as it was in the example above; it might be the domain of narrowly physical properties that I mentioned in section 1; or it might be the domain of natural properties - whatever you like. Now, one can ask of any object, what 'total conjunction' of these S-properties does that object exemplify? For example, in the simple case in which $\mathbb{S}$ contains only three properties, eight total conjunctions of $\mathbb{S}$-properties are possible - one example conjunction being the conjunction in which $S_{1}$ is exemplified but $S_{2}$ and $S_{3}$ are not exemplified. We can also ask for any total conjunction of $\mathbb{S}$-properties whether this conjunction is compatible with concept $a$. For example, since it is possible for there to be an object that is alkaline, and which 
at the same time exemplifies precisely those properties given by the conjunction contains hydroxide ions and does not contain ammonium ions, the latter conjunction is alkaline-compatible. More generally, to say that a conjunction is $a$-compatible is to say that it is possible for there to be an object that is [insert concept $a$ here] and at the same time exemplifies precisely those $\mathbb{S}$ properties given by this conjunction.

With these definitions in hand, let us make the following assumption:

(Necess) The following is absolutely necessary: whenever any object exemplifies precisely those properties given by any $a$-compatible total conjunction of $\mathbb{S}$-properties, then that object is [insert concept $a$ here].

For example, it is absolutely necessary that, whenever any object exemplifies those properties given by the conjunction contains hydroxide ions and does not contain ammonium ions, then that object is alkaline. In this respect, Necess says that this domain of $\mathbb{S}$-properties determines whether or not concept $a$ applies to any given object. In this respect, the latter concept supervenes on the former properties. (To be clear: to say that something is absolutely necessary is to say that it would be absurd if this were not the case. In other words, "the [absolutely] necessary is that whose negation counterfactually implies a contradiction" [Williamson 2007, 157]. See sec. 6 for discussion of some understandings of supervenience that do not appeal to absolute necessity.)

But let us also make the following assumption:

(Plenty Concepts) For any two concepts $x$ and $y$, there is a concept $x$ and $y$ that is their conjunction and a concept $x$ or $y$ that is their disjunction, and there is a concept that is their negation, not $x$, for example.

There is a concept, for example, that is the disjunction of the concept contains hydroxide ions and of the concept contains ammonium ions. One might call this concept the concept contains hydroxide or ammonium ions. To be more precise, it is a concept for which the following is knowable a priori: an object is [insert this concept] if and only if this object contains hydroxide ions or if this object contains ammonium ions.

It follows from Plenty Concepts that there is a concept that is the disjunction of all the $a$-compatible total conjunctions of $\mathbb{S}$-properties; call this concept $b$. (For example, at the beginning of this section, I took the concept contains hydroxide or ammonium ions to be the disjunction of all alkalinecompatible total conjunctions of microphysical properties.) But it follows from Necess that concept $b$ is necessarily co-applicable with concept $a .{ }^{5}$ By

5. See Kim (1984, 169-70; 1993, 151) and Bacon (1986) for proof. 
necessarily co-applicable I mean that it is absolutely necessary that any given object is [insert concept $a$ here] if and only if this object is [insert concept $b$ here].

But let us assume - temporarily for conditional proof - that concept $a$ denotes a property. Call this property $A$. And let us make the following assumption:

(Parity) If a concept denotes a property, then any necessarily co-applicable concept itself denotes some property.

So concept $b$ denotes some property too. Call this property $B$. So $B$ is necessarily coexemplified with $A$. But let us also make the following assumption:

(Property Taxonomy) Take any class of concepts, all of which denote properties of type $\mathbb{S}$. And take any concept that is formed purely out of taking conjunctions and disjunctions and negations of the concepts in this class. Then if this latter concept denotes a property, this property is itself of type $\mathbb{S}$.

For example, if a property is denoted by the concept contains hydroxide or ammonium ions, then this property is a microphysical property, because the concept contains hydroxide ions denotes a microphysical property and the concept contains ammonium ions denotes a microphysical property.

It follows that property $B$ is an $\mathbb{S}$-property. But let us assume that properties are individuated by necessary coexemplification:

(Indiv by Necess) If any property $P$ and any property $Q$ are necessarily coexemplified, then $P$ is the very same property as $Q$.

So property $A$ is the very same thing as $\mathbb{S}$-property $B$. So property $A$ is an S-property. We can now discharge our temporary assumption that concept $a$ denotes a property. This yields that if our given concept $a$ denotes a property, then this property is an S-property.

I do not suggest that the above argument should compel everyone to accept monism. Most of the assumptions in the above argument can be reasonably disputed. That said, section 5 will lend additional and independent support to Parity and sections 3 and 4 to Indiv by Necess. For the moment, however, I want to preempt some objections by clarifying three things.

Firstly, Plenty Concepts is very liberal about the existence of concepts. It issues in a concept is either negatively charged or made of iron, for example. But Plenty Concepts does not say that all concepts enjoy the same metaphys- 
ical status, as it were. Perhaps the concept is either negatively charged or made of iron does not denote a property, whereas the concept is made of iron does (Heil 2003). Or perhaps the concept is either negatively charged or made of iron does denote a property, but the property it denotes is not an objectively important property: it is a 'gerrymandered' or 'disunified' property, whereas is made of iron denotes a 'natural' or 'unified' property (Fodor 1974; Lewis 1983). Thus Plenty Concepts is compatible with the idea that some concepts (is either negatively charged or made of iron, e.g.) have an objectively inferior status to others (is made of iron, e.g.).

Second, my way of formulating the correlation argument does not specify the status of concept $a$ (the concept $i$ alkaline, e.g.). When my argument assumes that is alkaline denotes a property, it does so only temporarily for conditional proof. Thus my argument remains neutral about the existence or objective importance of 'multiply realizable' properties. Similarly, my argument does not specify the status of concept $b$ (the concept contains hydroxide or ammonium ions, e.g.). Thus my argument remains neutral about the existence or objective importance of 'disjunctive properties'. Instead, what my argument commits to is Parity, which is very roughly speaking the claim that the concept is alkaline denotes a property if and only if the concept contains hydroxide or ammonium ions denotes a property. It is of the utmost importance to note, however, that Parity on its own does not entail that the concept is alkaline denotes the same property as the concept contains hydroxide or ammonium ions, for example. Otherwise, assuming Parity would trivialize the correlation argument by making the other premises in the argument redundant. Instead, Parity merely entails that if the former concept denotes a property, then this latter concept denotes some property or other as well; see section 5 for discussion of this point. At any rate, Parity is a controversial thesis, I anticipate, and for this reason section 5 will lend additional and independent support to Parity.

Third, Plenty Concepts and Property Classification say things like concept $x$ or $y$ is the disjunction of concept $x$ and concept $y$. And in doing so you might think that Plenty Concepts and Property Classification are committed to there being some concepts that have the special status of being 'disjunctive' concepts. Not so. I defined the concept $x$ or $y$ to be a concept for which the following is knowable a priori: any given object is [insert concept $x$ or $y$ here] if and only if the object is [insert concept $x$ here] or the object is [insert concept $y$ here]. And, according to this definition, all concepts are disjunctions of some other concepts. The concept is negatively charged, for example, is a disjunction of the concept is negatively charged and in Australia and the concept is negatively charged and not in Australia. As a result, my argument sidesteps the tricky issue of what it even means for a property or concept to be disjunctive in any more substantial sense (Antony 1999; Clapp 2001; Shoemaker 2007; Audi 2013) — as it does the tricky issue of what it 
even means for a property or concept to be multiply realizable in any substantial sense (Shapiro 2000; Polger 2008).

3. Defending Individuation by Necessary Coexemplification. I have suggested that Antony's correlation argument is not as dialectically strong as it might be. The reason is that many dualists will be unsure about one of its premises, namely, that properties are individuated by necessary coexemplification (Indiv by Necess). For example, it looks like Shoemaker is committed to Indiv by Necess being true (McLaughlin 2009, sec. 2), but Marras (1993, 291) and Haug (2011a) and probably Fodor (1997) are committed to its being false. In the meta-ethics and meta-normativity literatures, Streumer (2008, sec. 4) reports that most dualists deny Indiv by Necess. This section therefore aims to lend force to the argument by showing how Indiv by Necess is supported on independent grounds.

One quick, independent way to motivate Indiv by Necess would be to point out that Indiv by Necess follows from the Lewisian ontology of properties. Consider a case in which, necessarily, any given individual exemplifies property $A$ if and only if it exemplifies $B$. It follows that exactly the same possible individuals exemplify $A$ that exemplify property $B$. But Lewis holds that properties just are these classes of possible individuals. So $A$ is the very same property as $B$. In short, Indiv by Necess: if property $A$ and property $B$ are necessarily coexemplified, then $A$ is the very same property as $B$.

But this quick argument for Indiv by Necess does not go very far. My experience is that dualists who are suspicious of Indiv by Necess are equally suspicious of the Lewisian ontology of properties. How then to lend force to Indiv by Necess? My strategy for lending credence to Indiv by Necess will be as follows. I will assume the following metaphysical thesis about the nature of singular causation:

(Difference Making) Event $C$ is a cause of event $E$ if and only if event $C$ makes a difference to event $E$.

Then on the basis of Difference Making, section 4 will argue for the following:

(Mirrored Causes) Whenever any properties (e.g., $P$ and $Q$ ) are necessarily coexemplified, then arbitrary event $\pi$ (associated with $P$ ) and arbitrary event $\psi$ (associated with $Q$ ) will have the same causes and effects.

But I will then make the following assumption:

(Powers Defined) If this arbitrary pair of events $\pi$ and $\psi$ have the same causes and effects, then the associated properties $P$ and $Q$ have the same causal powers. 
Monism Follows from

Follows from

Follows from

(I) Necess

(II) Plenty Concepts

(III*) Parity

(III) Boring Semantics

(IV) Property Taxonomy

Indiv by Necess $\longleftarrow\left\{\begin{array}{l}\text { (V) Indiv by Powers } \\ \text { (VI) Powers Defined } \\ \text { (VII*) Mirrored Causes }\end{array}\right.$

Sec. 2

Sec. 3

(VII) Difference Making

Figure 1.

Putting this together: whenever any properties (e.g., $P$ and $Q$ ) are necessarily coexemplified, then they have exactly the same causal powers. Next I will assume a principle of property individuation that is a putative alternative to Indiv by Necess:

(Indiv by Powers) Whenever any property $A$ and any property $B$ have exactly the same causal powers, then $A$ is the very same property as $B .^{6}$

Put differently, no two properties have exactly the same causal powers. So for property $P$ and property $Q$ in the above paragraph, it follows that $P$ is the same property as $Q$. In other words, Indiv by Necess follows: any necessarily coexemplified properties $(P$ and $Q)$ are the very same property. This is how I intend to lend credence to Indiv by Necess, a central premise in the correlation argument.

Therefore, the overall shape of the article is as follows. Section 2 showed how monism follows from five assumptions: (I) Necess, (II) Plenty Concepts, (III*) Parity, (IV) Property Taxonomy, and Indiv by Necess. But section 3 showed that Indiv by Necess in turn follows from three further assumptions: (V) Indiv by Powers, (VI) Powers Defined, and (VII*) Mirrored Causes. And section 4 will show that (VII*) Mirrored Causes in turn follows from (VII) Difference Making. And indeed section 5 will show that (III*) Parity in turn follows from (III) Boring Semantics. Thus, this article shows how monism follows from seven assumptions in total: I-VII. (This information is summarized in fig. 1.) Again, my aim is not to compel dualists to become monists, but to urge dualists to clarify their commitments, more on which in section 6 .

4. Mirrored Causes. In this section, I will assume Difference Making: singular causation is difference making. In fact, I will consider two specific ver-

6. See Armstrong (1978, sec. 16.1), Shoemaker (1980), Swoyer (1982), and Fodor (1987, 44-45). 
sions of Difference Making: singular causation is probability-raising (version 1), and singular causation is counterfactual dependence (version 2). My task will be to show that each of these versions of Difference Making issues in Mirrored Causes: whenever properties (e.g., $P$ and $Q$ ) are necessarily coexemplified, then the associated events $\pi$ and $\psi$ have the same causes and effects.

A warning: this probability-raising assumption and this counterfactual dependence assumption are very crude assumptions about the metaphysics of singular causation. These assumptions suffer from well-known objections, in particular, cases of causal preemption and overdetermination; and the probability-raising assumption also suffers from confounding via common causes (Collins, Hall, and Paul 2004). Nevertheless, the argument in this section can be modified to appeal to much more sophisticated versions of Difference Making, I contend. So you should think of the argument in this section as an idealized model, which can be fruitfully used to build a more sophisticated argument-depending on the precise nature of the more sophisticated version of Difference Making that you personally endorse. ${ }^{7}$

Another warning: the argument in this section will talk in the abstract about a property $P$ and a property $Q$. To fix ideas more concretely, it might help to imagine that $P$ is the property is alkaline and that $Q$ is the property contains hydroxide or ammonium ions. Of course, if you doubt that one or both of these properties exist, then you will need to choose a different example with which to make the argument in this section more concrete. It does not matter what example you choose: the argument of this section at no point appeals to any intuitions about any concrete cases. It proceeds on an entirely general and abstract level.

With this caveat in mind, take any given object that exemplifies any given property $P$ at any given time. For example, tomorrow this liquid will be alkaline. Let $\pi$ be the associated event, the event of the liquid being alkaline tomorrow, for example. Then consider any property $Q$ that is necessarily coexemplified with $P$. Take, for example, the property of containing hydroxide or ammonium ions. And consider the associated event $\psi$, for example, the event of this liquid containing hydroxide or ammonium ions tomorrow. In short, we will be considering simultaneous events $\pi$ and $\psi$, which are, re-

7. For example, you might endorse a more sophisticated way of treating causation as difference making. Something similar to what Halpern and Pearl (2005) and Halpern and Hitchcock (2015) propose: event $C$ was a cause of event $E$ if and only if there is some unspecified event $B$ such that $(a) B C>E$ and $(b) B \bar{C}>\bar{E}$, where $(c) C$ preceded $E$ and where $(d) B$ is such that there is a chain of dependence from singular variable $\mathbb{C}$ to singular variable $\mathbb{E}$, which does not go via singular variable $\mathbb{B}$. And one can, I contend, use the Restricted Transitivity (RT) and CSO axioms about counterfactual conditionals to establish that condition $d$, e.g., will hold for $\mathbb{C}=\pi$ if and only if condition $d$ holds for $\mathbb{C}=\psi$. 
spectively, associated with the exemplification of $P$ (being alkaline, e.g.) and of $Q$ (containing hydroxide or ammonium ions, e.g.) in the liquid tomorrow.

4.1. The Probability-Raising Principle. Let us start by assuming that singular causation is probability-raising. That is to say, event $C$ was a cause of event $E$ just in case $C$ preceded $E$, and $C$ increased the probability of $E$. And to say that $C$ increased the probability of $E$ is just to say that $P(E \mid C)>$ $P(E \mid \bar{C}) .^{8}$ This is mathematically equivalent to $P(E C)>P(E) P(C) .{ }^{9}$

The task in hand is to use the probability-raising principle to demonstrate Mirrored Causes. Let us start by observing that $P(\pi)=P(\psi)$. Indeed one can show that $P(E \pi)=P(E \psi)$ for any given event $E$. These claims follow from the wholly uncontroversial axiom of probability theory that impossible events have probability zero. ${ }^{10}$ It follows that $(a) P(E \pi)>P(E) P(\pi)$ is equivalent to $\left(a^{*}\right) P(E \psi)>P(E) P(\psi)$; just substitute the relevant terms. But $\pi$ and $\psi$ have the same temporal location, so $(b) \pi$ preceded $E$ if and only if $\left(b^{*}\right) \psi$ preceded $E$. Putting this together: $a$ and $b$ hold if and only if $a^{*}$ and $b^{*}$ hold. But the probability-raising principle says that $a$ and $b$ are equivalent to the claim that $\pi$ caused $E$; similarly, $a^{*}$ and $b^{*}$ are equivalent to the claim that $\psi$ caused $E$. Therefore, for any given event $E, \pi$ caused $E$ if and only if $\psi$ caused $E$. In other words, $\pi$ and $\psi$ have exactly the same effects.

Similarly recall that $P(\pi)=P(\psi)$ and $P(C \pi)=P(C \psi)$ for any given event $C$. It follows that $(a) P(C \pi)>P(C) P(\pi)$ is equivalent to $\left(a^{*}\right)$ $P(C \psi)>P(C) P(\psi)$; just substitute the relevant terms. But $\pi$ and $\psi$ have the same temporal location, so $(b) C$ preceded $\pi$ if and only if $\left(b^{*}\right) C$ preceded $\psi$. Putting this together, $a$ and $b$ hold if and only if $a^{*}$ and $b^{*}$ hold. But the probability-raising principle says that $a$ and $b$ are equivalent to the claim that $C$ caused $\pi$; similarly $a^{*}$ and $b^{*}$ are equivalent to the claim that $C$ caused $\psi$. Therefore, for any given event $C$, $\pi$ was caused by $C$ if and only if $\psi$ was caused by $C$. In other words, $\pi$ and $\psi$ have exactly the same causes.

The overall conclusion is Mirrored Causes: $\pi$ and $\psi$ have exactly the same causes and effects. The liquid's being alkaline tomorrow has exactly the same causes and effects as the liquid's containing hydroxide or ammonium ions tomorrow, to take just one concrete example (although recall my

8. I follow the mathematical convention of using overlines to denote the logical operation of negation. And $P(\mid)$ denotes conditional probability.

9. I follow the mathematical convention of using two conjoined letters such as $E C$ to represent the logical operation of conjunction.

10. By definition, $\pi$ and $\psi$ necessarily co-occur. So $\pi \bar{\psi}$ is impossible, as is $\psi \bar{\pi}$. So $Z \pi \bar{\psi}$ is impossible, as is $Z \psi \bar{\pi}$, for any given state of affairs $Z$. But it is an axiom of probability theory that impossible states of affairs have probability zero. So it is a theorem that $P(Z \pi \bar{\psi})=0$ and that $P(Z \psi \bar{\pi})=0$. But it is also a theorem of probability theory that $P(Z \pi)-P(Z \pi \bar{\psi})=P(Z \pi \psi)=P(Z \psi)-P(Z \psi \bar{\pi})$. By eliminating the zero terms we have $P(Z \pi)=P(Z \psi)$. And letting $Z$ be any tautology we also have $P(\pi)=P(\psi)$. 
caveat at the beginning of the section about whether this concrete example is well chosen).

4.2. The Counterfactual Dependence Principle. I will now argue for Mirrored Causes via an alternative route. I will assume an alternative version of Difference Making. This version says that singular causation is counterfactual dependence. That is to say, event $C$ was a cause of event $E$ just in case $C$ preceded $E$; and if $C$ had been absent, then $E$ would also have been absent (Lewis 1986). (For brevity I will express the latter counterfactual conditional formally as $\bar{C}>\bar{E}$.)

The task now is to use this counterfactual dependence principle to demonstrate Mirrored Causes. One can show that for any given event $E$, (a) $\bar{\pi}>\bar{E}$ holds if and only if $\left(a^{*}\right) \bar{\psi}>\bar{E}$ holds. This follows just from the CSO axiom governing conditionals. ${ }^{11}$ But $\pi$ and $\psi$ have the same temporal location, so $(b) \pi$ preceded $E$ if and only if $\left(b^{*}\right) \psi$ preceded $E$. Putting this together, $a$ and $b$ hold if and only if $a^{*}$ and $b^{*}$ hold. But the counterfactual dependence principle says that $a$ and $b$ are equivalent to the claim that $\pi$ caused $E$; similarly, $a^{*}$ and $b^{*}$ are equivalent to the claim that $\psi$ caused $E$. Therefore, for any given event $E, \pi$ caused $E$ if and only if $\psi$ caused $E$. In other words, $\pi$ and $\psi$ have exactly the same effects.

One can also show, however, that for any given event $C,(a) \bar{C}>\bar{\pi}$ holds if and only if $\left(a^{*}\right) \bar{C}>\bar{\psi}$ holds. This follows from the RT axiom governing conditionals. ${ }^{12}$ But $\pi$ and $\psi$ have the same temporal location, so $(b) C$ preceded $\pi$ if and only if $\left(b^{*}\right) C$ preceded $\psi$. Putting this together, $a$ and $b$ hold if and only if $a^{*}$ and $b^{*}$ hold. But the counterfactual dependence principle

11. Whenever one state of affairs such as $\psi$ necessitates another such as $\pi$, then we have $\bar{\pi}>\bar{\psi}$; and whenever $\pi$ also necessitates $\psi$, then we also have $\bar{\psi}>\bar{\pi}$. It follows that if $\bar{\pi}>\bar{E}$, then $\bar{\psi}>\bar{E}$, for any given state of affairs $E$. The reason is that the material conditional 'if $\bar{\pi}>\bar{\psi}$ and $\bar{\psi}>\bar{\pi}$, and if $\bar{\pi}>\bar{E}$, then $\bar{\psi}>\bar{E}$ ' is just an instance of the CSO axiom for conditionals (Nute and Cross 2002, 10, 87-88). By a similar logic we have: if $\bar{\psi}>\bar{E}$, then $\bar{\pi}>\bar{E}$. To grasp the $\mathrm{CSO}$ axiom consider the conditional $A>B$ : if Anil were to go to the party, then Beth would go to the party. And consider $B>A$ : if Beth were to go, then Anil would go. And $A>C$ : if Anil were to go, Charlie would go. And $B>C$ : if Beth were to go, then Charlie would go. The CSO axiom is the material conditional: if $A>B$ and $B>A$, and if $A>C$, then $B>C$ (Nute and Cross 2002, 10, 87-88).

12. Whenever one state of affairs such as $\psi$ necessitates another such as $\pi$, if $\pi$ were not to obtain, then $\psi$ would not either, no matter what other states of affairs also obtain. Therefore, we have $\bar{C} \bar{\pi}>\bar{\psi}$, for any given state of affairs $\bar{C}$. But the material conditional 'if $\bar{C} \bar{\pi}>\bar{\psi}$ and $\bar{C}>\bar{\pi}$, then $\bar{C}>\bar{\psi}$ ' is just an instance of the RT axiom for conditionals (Nute $1980,18,23)$. It follows that if $\bar{C}>\bar{\pi}$, then $\bar{C}>\bar{\psi}$. And by a similar logic we have: if $\bar{C}>\bar{\psi}$, then $\bar{C}>\bar{\pi}$. To grasp the RT axiom, consider the conditional $A>B$ : if Anil were to go to the party, then Beth would go to the party. And consider $A B>C$ : if Anil and Beth were to go, then Charlie would go. And consider $A>C$ : if Anil were to go, then Charlie would go. The RT axiom is the following material conditional: if $A>B$ and $A B>C$, then $A>C$. 
says that $a$ and $b$ are equivalent to the claim that $C$ caused $\pi$; similarly, $a^{*}$ and $b^{*}$ are equivalent to the claim that $C$ caused $\psi$. Therefore, for any given event $C, \pi$ was caused by $C$ if and only if $\psi$ was caused by $C$. In other words, $\pi$ and $\psi$ have exactly the same causes.

Again the overall conclusion is Mirrored Causes: $\pi$ and $\psi$ have exactly the same causes and effects. The liquid's being alkaline tomorrow has exactly the same causes and effects as the liquid's containing hydroxide or ammonium ions tomorrow, to take just one concrete example. Note that to support this conclusion, I did not appeal to anything as controversial as the Lewis-Stalnaker semantics for conditionals. I merely appealed to the RT and CSO axioms. And these axioms are relatively uncontroversial; see Nute $(1980,18,23,29,52,63,128-31)$ and also Nute and Cross $(2002,87-88)$. For example, these axioms hold even on Woodward's interventionist semantics for conditionals (Galles and Pearl 1998).

4.3. Summary. I have assumed, very crudely, that singular causation is probability-raising or counterfactual dependence. On this basis I have argued for Mirrored Causes: whenever any properties (e.g., $P$ and $Q$ ) are necessarily coexemplified, then arbitrary event $\pi$ (associated with $P$ ) and arbitrary event $\psi$ (associated with $Q$ ) will have the same causes and effects. The liquid's being alkaline tomorrow has exactly the same causes and effects as the liquid's containing hydroxide or ammonium ions tomorrow, to take one concrete example. The same conclusion also follows, I contend, for less crude versions of the assumption that singular causation is Difference Making. (At this point it is worth repeating the caveat at the beginning of this section that this concrete example may not be well chosen and also to repeat that the aim of the present article is not to establish monism conclusively.)

5. The Parity Thesis and 'The Property of' Locutions. I now turn my attention from Indiv by Necess to another controversial assumption of the correlation argument, namely, Parity. My aim in this section will be to lend some credence to Parity by showing that Parity follows from an extremely pedestrian thesis (Boring Semantics) about the semantics of 'the property of' locutions. It is not entirely unreasonable to deny Boring Semantics, I acknowledge, but section 6 will point out the costs of doing so.

Let us begin by noticing that monists and dualists often use locutions of the form 'the property of'- for example 'the property of being alkaline' or 'the property of containing either hydroxide or ammonium ions'. But what does it mean to talk of 'the property of being alkaline', for example? Here is a first attempt at an answer, which I will improve on in a moment. 'The property of being alkaline' denotes the property $X$ that meets the following conditions: $(1 a)$ the exemplification of property $X$ in any given object necessitates that the object is alkaline, and $(2 a)$ any given object being alkaline 
necessitates that the object exemplifies property $X$. (When I say that a state of affairs 'necessitates' a second state of affairs, I mean that it is absolutely impossible for the first state of affairs to obtain without the second obtaining also.) Of course, there may be no property that meets these conditions, in which case the locution 'the property of being alkaline' will fail to denote anything. Or indeed, there may be two or more properties $X_{1}$ and $X_{2}$ that meet these conditions, in which case, there must be some extra condition that functions as a tiebreaker, as it were. For example, one might think that 'the property of being alkaline' denotes the property out of $X_{1}$ and $X_{2}$ whose exemplification somehow 'grounds' the object being alkaline, in addition to necessitating that the object is alkaline (Fine 1994). Taking all this on board and generalizing away from the specific concept of being alkaline yields a more rigorous principle:

(Boring Semantics) 'The property of being [insert any concept $a$ here]' succeeds in denoting some property if and only if there is at least one property that meets the following conditions: (1) the exemplification of property $X$ in any given object necessitates that the object is [insert concept $a$ ], and (2) any given object being [insert concept $a$ ] necessitates that the object exemplifies property $X$.

In short, Boring Semantics is a very straightforward story about the conditions under which 'the property of' locutions succeed in denoting something (and a story that somewhat narrows down what these locutions denote, when they do denote). And as far as I am aware, the philosophical literature has not yet proposed any substantive alternatives to Boring Semantics.

This puts us in a position to argue for Parity. Let us suppose just for the moment that 'the property of being alkaline' succeeds in denoting a property. Boring Semantics then says that there is at least one property that meets both conditions ( $1 a$ ) and ( $2 a$ ) above. But let us also suppose just for the moment that, necessarily, any given object is alkaline if and only if it contains hydroxide or ammonium ions. So condition ( $1 a)$ above entails that $(1 b)$ the exemplification of property $X$ in any given object necessitates that the object contains hydroxide or ammonium ions; and condition $(2 a)$ above entails (2b) that any given object containing hydroxide or ammonium ions necessitates that the object exemplifies property $X$. The reason is that necessitation is a transitive relation. So, since conditions $(1 a)$ and $(2 a)$ above entail that conditions $(1 b)$ and $(2 b)$ are also met, and since there is at least one property that meets conditions $(1 a)$ and $(2 a)$, there is at least one property that meets conditions $(1 b)$ and $(2 b)$. But since there is at least one property that meets conditions $(1 b)$ and $(2 b)$, Boring Semantics says that the locution 'the property of containing hydroxide or ammonium ions' succeeds in denoting a property. Let us discharge our assumption that the locution 'the property of 
being alkaline' succeeds in denoting some property. This yields, if the 'the property of being alkaline' denotes some property, then 'the property of containing hydroxide or ammonium ions' denotes some property too. Let us also discharge our assumption that, necessarily, any given object is alkaline if and only if it contains hydroxide or ammonium ions. That is to say, let us discharge our assumption that the concept is alkaline is necessarily co-applicable with the concept contains hydroxide or ammonium ions. Discharging this assumption and expressing the conclusion in its most general form yields: (Parity): if a concept denotes a property, then any necessarily co-applicable concept itself denotes some property.

To preempt some objections, it is of the utmost importance to note the following point: Boring Semantics is consistent with there being two properties that satisfy conditions $(1 a)$ and $(2 a)$ and with there being two properties that satisfy conditions $(1 b)$ and $(2 b)$. In this case, the tiebreaker condition springs into action to make sure that 'the property of being alkaline' denotes a single property and 'the property of containing hydroxide or ammonium ions' denotes a single property. And for all Boring Semantics says, this tie-breaking condition may ensure that 'the property of being alkaline' denotes a property that is distinct from 'the property of containing hydroxide or ammonium ions'. Of course, the correlation argument will ultimately suggest that these two locutions each denote the very same property. Nevertheless, in assuming Boring Semantics, I do not beg the question here against the dualist by assuming at the outset that these two locutions each denote the same property. Put differently, Boring Semantics does not trivialize the correlation argument: it does not make the other premises in the argument redundant. ${ }^{13}$ (This same goes for Parity, which is just a direct consequence of Boring Semantics.)

In sum, to deny the Parity principle, the dualist would need to deny Boring Semantics, my extremely pedestrian understanding of 'the property of' locutions. This is not a wholly unreasonable thing to deny, I am happy to acknowledge. But its doing so does come with some costs: for one thing, it trivializes the dispute between correlation argument monists and dualists, as I will show in a moment.

6. Conclusion: Dualists Need to Be Clearer. The debate between dualists and monists is one of the central debates in the philosophy of mind, of biology, and of the social sciences. My aim in this article has not been to provide

13. Note also that Boring Semantics does not entail that if 'the property of being alkaline' denotes an objectively important property, then 'the property of containing hydroxide ions or ammonium ions' also denotes an objectively important property (sec. 2). And note also that Parity is a conditional thesis. So the above argument does not show that 'the property of containing hydroxide ions or ammonium ions' succeeds in denoting a property. 
decisive reasons for dualists to embrace monism - far from it. The aim instead has been to move the intricate debate between monists and dualists forward by sharply distinguishing the options available to the dualist.

Section 2 offered a new version of the correlation argument. Like Antony's version of this argument, my version has the virtue of being formulated as a deductive rather than as a nondeductive argument; contrast the arguments hinted at in Bacon (1986), Kim (1992), or Clapp (2001). And it also has the virtue of remaining neutral on the issue of the existence and objective importance of multiply realizable properties and disjunctive properties; contrast Clapp (2001) and Antony (2008). What's more, it has the virtue of making all its assumptions fully explicit. These assumptions are (I) Necess, (II) Plenty Concepts, (III*) Parity, and (IV) Property Taxonomy, as well as Indiv by Necess. And sections 3 and 4 strengthened the dialectical force of the correlation argument by showing that one of its controversial premises (Indiv by Necess) follows from some commonly accepted premises, namely, (V) Indiv by Powers, (VI) Powers Defined, and (VII) Difference Making. And section 5 strengthened its dialectical force even further, by showing that another controversial premise (Parity) follows from (III) Boring Semantics. In short, sections $2-5$ showed that dualists must reject at least one of the theses I-VII.

For a minority of dualists, it is already clear which of theses I-VII they would reject. Crane and Mellor (1990) reject Necess, for example, and dualists attracted by a hyperintensional approach to metaphysics would probably reject Difference Making (Jenkins and Nolan 2012; Nolan 2014), I suspect. However, for the majority of dualists it is entirely unclear which of I-VII they would reject.

Indeed, for each thesis from I to VII there are some dualists who are clearly committed to that thesis. First, I have already noted that most dualists endorse Necess, or at least seem to. Second, Indiv by Powers is the centerpiece of most dualists' metaphysical picture; see Fodor (1987, 44-45) and Shoemaker (2007), for example. Third, Fodor (1974, 1997), Pereboom and Kornblith (1991), Block (1997), Sawyer (2002), Haug (2011a, 2011b), and many other dualists are clearly committed to Plenty Concepts and to Parity and to Property Taxonomy. Indeed, they are happy to agree that there are an abundance of physical properties, for example, such as the property contains hydroxide or ammonium ions (Baker 1993, 81). Instead, these dualists insist that most of such properties are objectively unimportant; see section 2 and Antony (1999) for discussion.

Fourth, many dualists are committed to Difference Making: singular causation is difference making. To see this, note that Le Pore and Loewer (1987), Horgan (1989, 1997), Baker (1993), Bennett (2003), List and Menzies (2009), and many other dualists rely on Difference Making in order to undermine the exclusion principle at the heart of Kim's infamous exclusion argument. 
Kim's exclusion principle says that it is rare for a single event at time $t_{1}$ to have two distinct but 'complete' causes at time $t_{0}$. Therefore, a patient's experiencing a reduced level of pain, for example, was not caused both by the event of her ingesting codeine and also by the event of her ingesting an analgesic. But since the reduced level of pain was caused by the event of her ingesting codeine, it was not caused by the event of her ingesting an analgesic, Kim would argue. In order to reject Kim's conclusion here (and the exclusion principle on which it is based), the above dualists appeal to Difference Making: the event of ingesting an analgesic made a difference to her reduced levels of pain and therefore was a cause of it. Note that these dualists do not respond to Kim by claiming Difference Making*: difference making is sufficient for causation, but only if certain extra conditions hold (e.g., extra conditions: ingests an analgesic is an objectively important property; ingests an analgesic has the right sort of essence). After all, if these dualists were to rely only on Difference Making*, then they would have the extra burden of showing that these extra conditions do in fact hold in the case of the analgesic. And this would render their objection to Kim's exclusion principle dialectically ineffective and indeed utterly question begging.

In sum, for the majority of dualists it is entirely unclear which thesis of IVII they would reject; and for each thesis I-VII there are some dualists who are clearly committed to that thesis. My hope is to push the debate forward by putting pressure on dualists to be explicit about which of I-VII they will reject and why. I expect that doing so will uncover considerable heterogeneity among dualists.

7. The Costs of Dualism. I will finish up by reviewing the options available to the dualist. Dualists need to deny at least one of the following seven assumptions.

(Assumption I: Necess) The following is absolutely necessary: whenever any object exemplifies precisely those properties given by any $a$-compatible total conjunction of $\mathbb{S}$-properties, then this object is [insert concept $a$ here].

That is to say, concept $a$ supervenes on the S-properties (on the physical properties, e.g.). In my view, it is completely reasonable for monists and dualists to disagree over Necess. The disagreement here might stem from a comparatively trivial disagreement over what properties count as $\mathbb{S}$-type properties (what properties count as physical properties, e.g.). Or the disagreement might be more substantial. For example, a dualist might think that Necess holds only when 'absolutely necessary' is replaced with 'nomically necessary', the necessity associated with the laws of nature. If so, there are some generalizations that are nomically necessary (lawful) but that are not absolutely necessary. In that case, the dualist ought to say what exactly it is for 
a generalization to be nomically necessary (lawful) in the first place. What's more, she ought also to show that her reasons for endorsing Necess as a claim about nomic necessity are not themselves reasons for also endorsing Necess as a claim about absolute necessity. Either way, the dualist should make her reasons for denying Necess explicit.

(Assumption II: Plenty Concepts) For any two concepts $x$ and $y$, there is a concept $x$ and $y$ that is their conjunction and a concept $x$ or $y$ that is their disjunction, and there is a concept that is their negation, not $x$, for example.

As I have already discussed in section 2, the prospects for rejecting Plenty Concepts are not good. Of course, if one restricts one's attention to concepts that are humanly graspable, then Plenty Concepts will be false. But doing so risks turning this debate into a debate about subjective human limitations, not a debate about objective metaphysics (Clarke 2017).

(Assumption III: Boring Semantics) 'The property of being [insert any concept $a$ here]' succeeds in denoting some property if and only if there is at least one property that meets the following conditions: (1) the exemplification of property $X$ in any given object necessitates that the object is [insert concept $a$ here], and (2) any given object being [insert concept $a$ here] necessitates that the object exemplifies property $X$.

Section 5 has explained why Boring Semantics is much less controversial than it may first appear. Note also that Boring Semantics may be true for some users of 'the property of' locutions and false for other users. Indeed, as far as monist users are concerned, Boring Semantics makes sense of everything that monists say. So the most charitable interpretation of such monists is that Boring Semantics is true of monist uses of 'the property of' locutions. Consider then a dualist who concedes that Boring Semantics is true for monist uses of 'the property of' locutions, but who insists that it is false for dualist uses. To some extent, this is a reasonable move for the dualist to make. But this move would radically reconfigure how the monist versus dualist debate is typically framed: the debate becomes a semantic debate in which the monist and dualist are talking past each other by using 'the property of' locutions in different ways. Furthermore, this move burdens dualists with the following challenge: how is the reference of 'the property of' locutions determined for dualist users, if not by Boring Semantics? To the extent that dualists cannot give a substantive answer to this question, dualism is a less explanatory theory, one that creates a mystery where there need be no mystery.

(Assumption IV: Property Taxonomy) Take any class of concepts, all of which denote properties of type $\mathbb{S}$. And take any concept that is formed 
purely out of taking conjunctions and disjunctions and negations of the concepts in this class. Then if this latter concept denotes a property, this property is itself of type $\mathbb{S}$.

For example, if a property is denoted by the concept contains hydroxide or contains ammonium ions, then this property is a physical property, because the concept contains hydroxide ions denotes a physical property and the concept contains ammonium ions denotes a physical property. As far as I can see, Property Taxonomy is the only way of justifying or explaining - in a systematic and principled manner - why paradigm physical properties deserve to be counted as physical. Take, for example, the property has kinetic energy of less than 10 joules or the property is hydrocodone or ibuprofen or codeine; see Clarke (2017) for discussion. Nevertheless, it is completely reasonable for dualists to disagree with Property Taxonomy, I think. But such dualists should acknowledge that, in doing so, they are jettisoning a more systematic and principled approach to classifying properties as physical in favor of a more intuitive and less systematic approach. At any rate, denying Property Taxonomy makes the disagreement between dualists and 'correlation argument monists' a comparatively superficial disagreement over what properties to label as physical properties, for example. So, once this has been revealed to be the fundamental point of disagreement between a monist and a dualist, then there is no point in their pursuing this disagreement further, I urge. If so, why has this point (that the disagreement is a trivial one over Property Taxonomy) not been recognized sooner? one might ask. Here the blame lies more with dualists, I think. After all, Property Taxonomy has been a well-advertised feature of many monists' philosophies (Kim 1998, 82). ${ }^{14}$

(Assumption V: Indiv by Powers) Whenever property $P$ and property $Q$ have exactly the same causal powers, then $P$ is the very same property as $Q$.

To some extent, it is reasonable for monists and dualists to disagree over Indiv by Powers, I think. But denying Indiv by Powers introduces mysteries where there need be none, namely, about how properties are individuated. And it also creates some corresponding epistemological problems for the dualist. In the absence of some alternative principle about how to individuate properties, how come you are confident that mental properties are not physical properties, for example, or that normative properties are not natural properties? Blind faith alone? Or because you are unwittingly assuming that no two concepts denote the same property? If the latter, then this trivializes the

14. I suspect that McLaughlin (2009, sec. 2) makes this criticism of Shoemaker's version of dualism. 
debate: questions about property identity are no longer questions about objective metaphysics, but are instead questions about human concepts (Clarke 2017).

(Assumption VI: Powers Defined) If an arbitrary pair of events $\pi$ and $\psi$ have the same causes and effects, then the associated properties $P$ and $Q$ have the same causal powers.

I myself can see no motivation for denying this assumption.

(Assumption VII: Difference Making) Event $C$ is a cause of event $E$ if and only if $C$ makes a difference to $E$.

Here difference making can be defined crudely in terms of probabilityraising or counterfactual dependence, or it can be given a more sophisticated definition; see Halpern and Hitchcock (2015), for example. At any rate, it is reasonable for dualists to deny that there is any suitable definition of difference making for which Difference Making is true. But doing so introduces mysteries where there need be none, namely, about the metaphysics of singular causation. And it creates some corresponding epistemological problems for the dualist: Why do you think that mental events can cause other events? How do scientists come to know what causes what, other than by examining difference making? Causal inference is difficult enough without philosophers introducing additional and unwelcome obstacles.

\section{REFERENCES}

Antony, Louise M. 1999. "Multiple Realizability, Projectability, and the Reality of Mental Properties." Philosophical Topics 26:1-24.

- 2008. "Multiple-Realization: Keeping It Real." In Being Reduced: New Essays on Reduction, Explanation and Causation, ed. Jakob Hohwy and Jesper Kallestrup, 164-75. Oxford: Oxford University Press.

Armstrong, David M. 1978. A Theory of Universals. Cambridge: Cambridge University Press.

Audi, Paul. 2013. "How to Rule Out Disjunctive Properties." Noûs 47:748-66.

Bacon, John. 1986. "Supervenience, Necessary Coextensions, and Reducibility." Philosophical Studies 49:163-76.

Baker, Lynne Rudder. 1993. "Metaphysics and Mental Causation.” In Mental Causation, ed. John Heil and Alfred R. Mele, 75-96. Oxford: Oxford University Press.

- 2007. The Metaphysics of Everyday Life: An Essay in Practical Realism. Cambridge: Cambridge University Press.

Bennett, Karen. 2003. "Why the Exclusion Problem Seems Intractable and How, Just Maybe, to Tract It." Noûs 37:471-97.

Block, Ned. 1997. “Anti-reductionism Slaps Back.” Philosophical Perspectives 11:107-32.

Chalmers, David J. 1996. The Conscious Mind: In Search of a Fundamental Theory. Oxford: Oxford University Press.

Clapp, Lenny. 2001. "Disjunctive Properties: Multiple Realizations." Journal of Philosophy 98:111-36. 
Clarke, Christopher. 2016. "The Explanatory Virtue of Abstracting Away from Idiosyncratic and Messy Detail." Philosophical Studies 173:1429-49.

—. 2017. "How to Define Levels of Explanation and Evaluate Their Indispensability." Synthese 194:2211-31.

Collins, John, Ned Hall, and L. A. Paul, eds. 2004. Causation and Counterfactuals. Cambridge, MA: MIT Press.

Crane, Tim, and D. H. Mellor. 1990. "There Is No Question of Physicalism.” Mind 99:185206.

Fine, Kit. 1994. "Essence and Modality.” Philosophical Perspectives 8:1-16.

Fodor, Jerry. 1974. "Special Sciences (or the Disunity of Science as a Working Hypothesis)." Synthese 28:97-115.

- 1987. Psychosemantics: The Problem of Meaning in the Philosophy of Mind. Cambridge, MA: Bradford/MIT Press.

1997. "Special Sciences: Still Autonomous after All These Years." Philosophical Perspectives 11:149-63.

Galles, D., and Judea Pearl. 1998. "An Axiomatic Characterization of Causal Counterfactuals." Foundations of Science 3:151-82.

Halpern, Joseph Y., and Christopher Hitchcock. 2015. "Graded Causation and Defaults." British Journal for the Philosophy of Science 66:413-57.

Halpern, Joseph Y., and Judea Pearl. 2005. "Causes and Explanations: A Structural-Model Approach. Part I: Causes.” British Journal for the Philosophy of Science 56:843-87.

Haug, Matthew C. 2011a. "Abstraction and Explanatory Relevance; or, Why Do the Special Sciences Exist?” Philosophy of Science 78:1143-55.

_. 2011b. "Natural Properties and the Special Sciences." Monist 94:244-66.

Heil, John. 2003. "Levels of Reality." Ratio 16:205-21.

Heil, John, and David Robb. 2003. "Mental Properties." American Philosophical Quarterly 40:175-96.

Horgan, Terence E. 1989. "Mental Quausation.” Philosophical Perspectives 3:47-74.

- 1997. "Kim on Mental Causation and Causal Exclusion." Philosophical Perspectives 11:165-84.

Hüttemann, Andreas. 2004. What's Wrong with Microphysicalism? London: Routledge.

Jackson, Frank. 2002. "Review: From Reduction to Type-Type Identity." Philosophy and Phenomenological Research 65:644-47.

Jaworski, William. 2002. "Multiple-Realizability, Explanation and the Disjunctive Move." Philosophical Studies 108:289-308.

Jenkins, C. S., and Daniel Nolan. 2012. "Disposition Impossible.” Nô̂s 46 (4): 732-53.

Kim, Jaegwon. 1984. "Concepts of Supervenience.” Philosophy and Phenomenological Research 45:153-76.

—. 1992. "Multiple Realization and the Metaphysics of Reduction." Philosophy and Phenomenological Research 52:1-26.

—. 1993. "Supervenience as a Philosophical Concept." In Supervenience and Mind, 131-60. Cambridge: Cambridge University Press.

1998. Mind in a Physical World. Cambridge, MA: MIT Press.

Le Pore, Ernest, and Barry Loewer. 1987. "Mind Matters." Journal of Philosophy 84:630-42.

Lewis, David K. 1966. "An Argument for the Identity Theory." Journal of Philosophy 63:17-25.

- 1983. "New Work for a Theory of Universals." Australasian Journal of Philosophy 61:343-77.

—. 1986. "Causal Explanation.” In Philosophical Papers, vol. 2, 214-40. Oxford: Oxford University Press.

—. 1994. "Reduction of Mind." In A Companion to Philosophy of Mind, ed. Samuel Guttenplan, 412-31. Oxford: Blackwell.

List, Christian, and Peter Menzies. 2009. "Non-reductive Physicalism and the Limits of the Exclusion Principle." Journal of Philosophy 106:475-502.

Macdonald, Cynthia, and Graham Macdonald. 2006. "The Metaphysics of Mental Causation." Journal of Philosophy 103:539-76.

Macdonald, Graham. 1992. "Reduction and Evolutionary Biology." In Reduction, Explanation and Realism, ed. David Charles and Kathleen Lennon, 69-96. Oxford: Oxford University Press. 
Marras, Ausonio. 1993. "Psychophysical Supervenience and Nonreductive Materialism." Synthese 95:275-304.

McLaughlin, Brian P. 2007. "Type Materialism for Phenomenal Consciousness.” In The Blackwell Companion to Consciousness, ed. M. Velmans and S. Schneider, 431-44. London: Blackwell. . 2009. Review of Physical Realization, by Sydney Shoemaker. Notre Dame Philosophical Reviews 2009 (7).

Mellor, D. H. 1982. "The Reduction of Society." Philosophy 57:51-75. . 2008. "Micro-Composition." Royal Institute of Philosophy Supplement 83:65-80.

Ney, Alyssa. 2008. "Defining Physicalism." Philosophy Compass 3:1033-48.

Nolan, Daniel. 2014. "Hyperintensional Metaphysics." Philosophical Studies 171:149-60.

Nute, Donald. 1980. Topics in Conditional Logic. Dordrecht: Reidel.

Nute, Donald, and C. B. Cross. 2002. "Conditional Logic.” In Handbook of Philosophical Logic, vol. 4, rev. 2nd ed., ed. Don M. Gabbay and F. Guenthner, 1-98. Dordrecht: Kluwer.

Papineau, David. 2001. "The Rise of Physicalism." In Physicalism and Its Discontents, ed. Carl Gillett and Barry Loewer, 3-36. Cambridge: Cambridge University Press.

Pereboom, Derk, and Hilary Kornblith. 1991. "The Metaphysics of Irreducibility." Philosophical Studies 63:125-45.

Pettit, Philip. 1993. The Common Mind. Oxford: Oxford University Press.

Polger, Thomas. 2008. "Two Confusions Concerning Multiple Realization." Philosophy of Science 75:537-47.

Rosenberg, Alex. 2006. Darwinian Reductionism. Chicago: University of Chicago Press.

Ruben, David-Hillel. 1984. "Social Properties and Their Basis." Proceedings of the Aristotelian Society 85:23-45.

Sawyer, R. Keith. 2002. "Nonreductive Individualism: Part I-Supervenience and Wild Disjunction." Philosophy of the Social Sciences 32:537-59.

Shapiro, Lawrence A. 2000. "Multiple Realizations.” Journal of Philosophy 97:635-54.

Shoemaker, Sydney. 1980. "Causality and Properties." In Time and Cause: Essays Presented to Richard Taylor, ed. P. van Inwagen, 109-35. Dordrecht: Reidel.

- 2001. "Realization and Mental Causation." In Physicalism and Its Discontents, ed. Carl Gillett and Barry Loewer, 74-98. Cambridge: Cambridge University Press. 2007. Physical Realization. Oxford: Oxford University Press.

Smart, J. J. C. 1959. "Sensations and Brain Processes.” Philosophical Review 68:141-56.

Streumer, Bart. 2008. "Are There Irreducibly Normative Properties?” Australasian Journal of Philosophy 86 (4): 537-61.

Swoyer, Chris. 1982. "The Nature of Natural Laws." Australasian Journal of Philosophy 60:20323.

Williamson, Timothy. 2007. The Philosophy of Philosophy. Oxford: Blackwell. 